\title{
(f) La Arqueología del \\ Paisaje como programa de gestión integral del Patrimonio Arqueológico
}

En un trabajo anterior propusimos reformular la gestión del Patrimonio Histórico como una práctica interpretativa, como una práctica que moviliza y produce valores intelectuales que tienen elementos físicos concretos como referentes. Argüimos asímismo que esa formulación permitiría integrar la investigación y administración del Patrimonio Histórico y superar la situación de fragmentación y multiplicidad que predomina actualmente en la gestión de ese Patrimonio que, como mínimo, diferencia, de un modo a menudo irreconciliable, entre labores de estudio, administración, divulgación e intervención directa. Todas ellas, en cambio, pueden ser subsumidas dentro de un modelo de gestión unitario concebido como una cadena valorativa o, mejor, encadenamiento de valoraciones sucesivas que poseen los mismos referentes, pero se orientan hacia diferentes objetos y terminan por producir resultados distintos.

En esta colaboración pretendemos ir dos pasos más allá. De un modo que intenta ser coherente con la reorganización de las prácticas arqueológicas según la cadena valorativa y, asímismo, con el proyecto de reconversión de la Arqueología en Tecnología, intentaremos explorar la funcionalidad práctica de estas propuestas y, al tiempo, proponer un modelo de gestión del Patrimonio Arqueológico fundado sobre un programa de investigación en Arqueología del Paisaje. Evidentemente no es la única alternativa posible, ni pretende ser mejor que otras. Es la que a nosotros se nos ocurre $y$, desde nuestro punto de vista, presenta varias ventajas que veremos a continuación.

\section{De la Arqueología de urgencia a la investigación arqueológica}

Hace ahora cuatro años, en octubre de 1991, la Dirección Xeral do Patrimonio Histórico e Documental de la Consellería de Cultura de la Xunta de Galicia solicitó a la Universidad de Santiago de Compostela que se encargase de la dirección y coordinación de los proyectos de control del Impacto Arqueológico de las grandes Obras
Públicas que se iban a desarrollar durante los años siguientes en nuestro país. Estas Obras se agrupaban esencialmente en dos conjuntos bien definidos: por una parte están el Oleoducto Coruña-Vigo promovido por CLH y la Red de Gasificación de Galicia, proyectada por ENAGAS, y por otra las Autovías Benavente-Lugo-Coruña y Benavente-Ourense-Vigo del MOPT.

El Departamento de Historia I de la USC asumió ese encargo y, por su parte, responsabilizó del desarrollo del mismo al firmante de este artículo. Para satisfacer estas demandas se constituyó un grupo universitario de investigación. Los trabajos se han realizado en colaboración con la Dirección Xeral do Patrimonio Histórico e Documental y con las Empresas promotoras, proyectistas o de construcción responsables de las obras. Esta línea de acción está auspiciada por la política en I+D que sigue la Universidad española (reconocida y propiciada por la Ley de Reforma Universitaria) y, en concreto, por la Universidad de Santiago (según ha sido regulado en sus Estatutos). El instrumento garante y coordinador de esta política de cooperación con la Empresa y otros servicios de la Administración es, concretamente, el Centro de Transferencia de Tecnología de la USC, que se ocupa de oficializar esas colaboraciones.

Al mismo tiempo, llevados por el incremento de la demanda de acciones de puesta en valor del Patrimonio Arqueológico, tuvimos la ocasión de diseñar diversos planes y proyectos de rentabilización socio-cultural de ese Patrimonio. Este ámbito reune la realización de: Planes Directores para yacimientos o parajes arqueológicos, proyectos de ordenación de recursos para ayuntamientos, exposiciones arqueológicas, asesoramientos y estudios básicos para plantear programas de musealización, consolidación o protección de monumentos y bienes arqueológi-

\section{Felipe Criado Boado}

Grupo de Trabajo de Arqueología del Paisaje Departamento de Historia 1 Universidad de Santiago de Compostela 




cos, etc... En concreto, se realizó el Plan Director para la rentabilización socio-cultural del Patrimonio Arqueológico del Ayuntamiento de Toques, (Coruña) y se acometieron otros trabajos del mismo estilo y menor porte.

La intervención directa de la Universidad en estos contextos, tradicionalmente reservados a empresas de Arqueología y arqueólogos liberales, puede resultar muy discutible. Con todo, y a pesar de que no es muy frecuente dentro de las Humanidades, representa una línea de acción bien conocida y justificada en otros ámbitos de la investigación universitaria y de la colaboración Universidad-Empresa. Como en cualquiera de estos otros casos, lo que legitima la introducción de la universidad en este mundo es la necesidad de: (a) establecer los criterios y metodologías de intervención en un campo de trabajo inédito, (b) formar los recursos humanos para actuar en él y (c), en definitiva, contribuir a la consolidación de este área de actividad. En síntesis, se trataba de readaptar la disciplina arqueológica para poder cubrir demandas sociales concretas $y$, en definitiva, para satisfacer una función tecnológica.

Después de cuatro años de experiencia, creemos que la Universidad española tiene el desafío y la posibilidad de comprometerse de forma activa en estas funciones. Es cierto que los problemas prácticos son muchos, que el ambiente universitario y exterior no facilita este tipo de acciones y que el propio sistema universitario es, en muchos casos, demasiado rígido para poderse adaptar con flexibidad a estas necesidades. Por todo ello, el éxito de este tipo de empresas depende en gran medida de la existencia (como ocurrió en nuestro caso) de una intención decidida y un apoyo concreto por parte de la estructura universitaria, tanto por parte de los departamentos de Arqueología y Prehistoria, como de los órganos de decisión y representación. Además se precisa una voluntad clara por parte de la administración del Patrimonio Arqueológico, que debe disponer de una idea muy bien definida de la función que la universidad debe jugar en estos contextos. Por último, es necesario el consenso de todos los sectores que intervienen sobre el Patrimonio Arqueológico: debe quedar claro para todas las partes que el papel de la universidad no es competir con el sector privado en su terreno de juego sino, como indicábamos más arriba, desarrollar las aplicaciones metodológicas (tecnológicas, diríamos) para actuar en él. Esta función terminará, a corto plazo, repercutiendo favorablemente sobre el propio sector privado $y$, sobre todo, beneficiará a todas las partes al contribuir de este modo a la vertebración de un área de trabajo tan invertebrada como la Arqueología. En concreto, esta colaboración permitiría superar la manida y destructiva oposición entre investigación y gestión y haría que la universidad, sin ceder un ápice en sus objetivos primarios, se comprometiera con las prioridades de la administración del Patrimonio Arqueológico.
Sin embargo, el problema fundamental de esta línea de acción es alejarse de su estricta dimensión empírica y, en cambio, ser capaces de orientar el trabajo en ella desde una perspectiva cognitiva y crítica más amplio. Este tipo de actividades deben converger en y, es más, ser orientadas desde un programa de investigación concreto. Sólo éste puede aportar el planteamiento, la metodología, las hipótesis de trabajo, las convenciones de registro, los modelos interpretativos e, incluso, los criterios de acción práctica, para poder satisfacer las demandas concretas $y$, sin embargo, ser capaces de remontarse sobre lo particular para apuntar hacia objetivos más generales.

De hecho, el trabajo que nuestro grupo realiza en todas esas áreas de actividad, se concreta fundamentalmente en tres ámbitos complementarios:

1. Desarrollo de los planteamientos teóricos y procedimientos metodológicos para intervenir en ellos desde la ArPa.

2. Registro de la información recogida en los trabajos prácticos realizados.

3. Utilización de esta información para generar interpretaciones y reconstrucciones del pasado arqueológico que puedan, en definitiva, retroalimentar el sistema de trabajo.

Es necesario que la filosofía de trabajo prime el desarrollo simultáneo y armónico de los tres ámbitos anteriores. En la medida en que cada uno de ellos sea adecuadamente enfocado, era imprescindible interrelacionar en todo momento el trabajo técnico efectivo y las acciones de investigación.

Como ya se ha dicho, la ArPa es el programa de investigación o estrategia cobertora que, en nuestro caso, orienta el trabajo concreto. Nuestro esfuerzo empezó siendo el de un equipo de investigación que utilizaba datos y recursos procedentes de asistencias técnicas para realizar estudios de los paisajes arqueológicos; siguió siendo el de un grupo de trabajo que, basándose en un programa de investigación específico, desarrolló las metodologías precisas para trabajar en esos trabajos; y terminó por ser el de una unidad de trabajo que afronta todas las fases de valoración del Patrimonio Arqueológico adaptando un modelo de gestión integral de ese Patrimonio que se concreta en la cadena valorativa y se proyecta como una Arqueología del Paisaje.

En lo que resta de este comentario intentaremos mostrar la utilidad de la ArPa en este contexto.

\section{Hacia una Arquelogía del Paisaje}

M. Godelier (Lo ideal y lo material, trad. 1989) propuso que el ser humano, a diferencia de otros seres vivos, no sólo vive en el entorno, sino que crea su propio entorno para vivir o, dicho en otras palabras, construye su propio medio socio-cultural. Este principio es útil para fundar un estudio arqueológico del paisaje social. De hecho, aunque la artificialización progresiva del medio, se ha 
acelerado en la historia reciente de la Humanidad, este fenómeno no es exclusivo de las sociedades industriale, ni de las organizaciones sociales complejas y ni tan siquiera de la generalización (hace más de 7000 años) del modo de vida agrícola. Este fenómeno es antes bien una característica básica que define qué es la Humanidad y diferencia a ésta de otras formas de vida.

El estudio de este fenómeno desde el punto de vista de la Arqueología, constituye la Arqueología del Paisaje. Dentro de ella es necesario definir de forma explícita lo que se entiende por paisaje. Yendo más allá de la concepción empirista de éste, que lo entiende como una realidad ya dada y que, por diferentes razones, se niega a sí misma, y de la sociológica, que explica el paisaje como el medio y el producto de los procesos sociales, nosotros proponemos concebir el paisaje como el producto socio-cultural creado por la objetivación, sobre el medio y en términos espaciales, de prácticas sociales tanto de carácter material como imaginario.

Complementariamente, podríamos definir la Arqueología del Paisaje como una estrategia de investigación que pretende reconstruir e interpretar los paisajes arqueológicos a partir de los objetos que los concretan. Esta declaración genérica quiere decir, en un sentido más práctico, que la ArPa es la inclusión de la práctica arqueológica dentro de coordenadas espaciales. A través de ella se trata de pensar el registro arqueológico y la cultura material desde una matriz espacial y, simultáneamente, de convertir al espacio en objeto de la investigación arqueológica.

En este sentido, la ArPa constituye más un manifiesto sobre la forma de hacer Arqueología, que una orientación o escuela específica de la disciplina. En otros puntos hemos intentado establecer la posición teórica y práctica de la ArPa, según la definimos y aplicamos nosotros. Sus principales características serían, expuestas de un modo sintético y progresivo, las siguientes:

I. El tratamiento de los elementos del registro arqueológico como entidades formales que son el producto 0 efecto de la acción social;

2. el reconocimiento de que esas entidades sociales, siendo formas, se proyectan espacialmente $y$, por lo tanto, son visibles, esto es: pueden ser percibidas y descritas por la observación arqueológica;

3. la asunción de que esas entidades son el resultado de un determinado pliegue o inflexión entre el mundo efectivo y el universo conceptual que lo posibilita;

\section{4. la presuposición de que todo lo visible es simbólico;}

5. la utilización de las condiciones de visibilidad del registro arqueológico como recurso de descripción e interpretación del mismo;

6. la definición del contexto espacial del registro arqueo- lógico, tanto actual como original, como método de análisis del mismo;

7. la reconstrucción de su contexto original buscando relaciones espaciales significativas entre las entidades del registro arqueológico o sus proyecciones con otros códigos o niveles de su misma formación social;

8. la utilización de una descripción densa, basada sobre todo en el análisis formal como procedimiento de trabajo, que permita aprehender los fenómenos considerados sin introducir un sentido extraño a ellos;

9. y la interpretación del significado del contexto espacial del registro arqueológico mediante analogías débiles entre éste y el paisaje actual (o tradicional) en el que se aparece.

\section{Aplicaciones de la Arqueología del Paisaje}

La ArPa como línea de investigación comprende el estudio y reconstrucción de los paisajes arqueológicos o, mejor, el estudio con metodología arqueológica de los procesos y formas de culturización del espacio a través de la historia. En este sentido, tiene el doble objetivo de:

I. Reconstruir los paisajes sociales de épocas prehistóricas $y$,

2. estudiar los procesos de cambio y continuidad que han constituido el paisaje rural actual.

Aunque la ArPa es compatible con la Landscape Archaeology británica, se diferencia de esta línea de investigación que, en muchos casos, es en realidad una Arqueología Rural y, por lo tanto, parte del segundo objetivo que se acaba de enumerar. En cambio, la estrategia de trabajo que nosotros entendemos como ArPa implica analizar las tres dimensiones que, de forma complementaria, constituyen el paisaje:

A. El espacio en cuanto entorno físico o matriz medioambiental de la acción humana. Este trabajo sólo puede ser abordado desde la colaboración de la Arqueología con disciplinas medioambientales; la paleoecología y la geoarqueología ofrecen el marco básico para considerar esta dimensión.

B. El espacio en cuanto entorno social o medio construido por el ser humano en el que se producen las relaciones entre individuos y grupos.

C. El espacio en cuanto entorno simbólico o medio pensado que ofrece la base para desarrollar, y comprender, la apropiación humana de la 
naturaleza.

Desde esta triple dimensión, la ArPa comprende el estudio de todos los procesos sociales e históricos en su dimensión espacial y se orienta, entre otras cosas, hacia el estudio del poblamiento prehistórico e histórico, el análisis de las formas de utilización y modificación del medio, la caracterización del efecto humano sobre el medio natural y la reconstrucción de éste. Un horizonte específico de análisis es el estudio de las formas y estructuras de parcelación y uso del campo (terrazas agrícolas, bancales, parcelas, sistemas de riego...). Esta temática es especialmente significativa en un país como Galicia, dotado de un sistema agrario tradicional cuyo espesor histórico y permanencia ha dado lugar a un paisaje humanizado que, en su apariencia actual, conserva elementos antiguos.

Sin embargo, más allá de los horizontes y dimensiones temáticas específicas de la ArPa, ésta posibilita generar una línea de aproximación que posee implicaciones relevantes en todos los ámbitos del registro y Patrimonio Arqueológico. En concreto, y siguiendo una escala progresiva desde la investigación de carácter básico a la aplicada, desde la producción de conocimiento nuevo a la administración de las entidades arqueológicas, los principios y metodologías propios de la ArPa aportan una base eficaz no sólo para estudiar el registro arqueológico de una forma más plena, sino también para: (a) desarrollar planteamientos teóricos y procedimientos metodológicos para intervenir en el Patrimonio Arqueológico, (b) solventar problemáticas concretas de ese Patrimonio, y (c) satisfacer las nuevas funciones que la sociedad plantea a la Arqueología.

Esta estrategia de trabajo facilita la resolución de muchos problemas prácticos vinculados al Patrimonio Arqueológico por cuanto éstos tienen un inevitable carácter espacial. En la actualidad se tiende cada vez más a, como sancionó la convención Heritages for Europe convocada por el Consejo de Europa en septiembre de 1994, ampliar el concepto de Patrimonio para comprender desde un elemento histórico concreto hasta el conjunto del paisaje como entorno construido y huella de la humanidad. Esto subraya ya no la conveniencia, sino la necesidad de poner a punto estrategias de investigación y evaluación del registro arqueológico que reintroduzcan a éste en su matriz espacial. Al fundir ese registro con el ambiente espacial al que pertenece, se facilita la interpretación, diagnóstico y administración tanto del uno como del otro.

\section{La cadena valorativa en Arqueología del Paisaje}

Así pues, la ArPa no constituye simplemente una línea de investigación, sino que representa una perspectiva rentable para diseñar y desarrollar un plan de gestión integral del Patrimonio Arqueológico que cumpla las condiciones enunciadas en nuestro anterior comentario. En concreto, permite plantear y articular todas las fases de valoración y revalorización del Patrimonio Arqueológico que se desarrollan dentro de la Arqueología concebida como cadena interpretativa. Veremos a continuación cómo, utilizando los parámetros de la ArPa, se pueden modificar los sistemas, técnicas y criterios de trabajo en Arqueología.

\section{Recuperación}

Las intervenciones arqueológicas (la prospección y la excavación fundamentalmente) se pueden redefinir basándose en el hecho obvio de que son actuaciones de carácter territorial (que tienen el territorio por base y, a veces, objetivo). Además de ello, la consideración de variables espaciales precisa reformular la concepción y ejercicio de esas actuaciones. Sin embargo, en este sentido ya se ha avanzado mucho gracias a las aportaciones de la Arqueología del Territorio.

\section{Valoración}

En cambio, es más urgente redefinir las entidades arqueológicas para acomodarlas a la perspectiva que incorpora la ArPa. Esta, en realidad, se corresponde con la línea de acción que se abre en Arqueología cuando se asume como principio esencial el hecho de que las entidades arqueológicas (yacimientos, materiales, estructuras...) no existen aisladas, sino que son entidades espaciales, relacionadas significativamente con su contexto espacial y comprensibles (e incluso predecibles) desde esta dimensión.

Así, por ejemplo, deberemos reformular el concepto de yacimiento arqueológico: éste no es una entidad aislada y autónoma, sino el eje (o núcleo) de un paisaje artificial prehistórico del que depende y al que representa. Igualmente, el estudio del registro arqueológico, desde el análisis de la cultura material arqueológica (cerámica, adorno, escultura...), hasta la distribución del poblamiento y el uso del suelo, pasando por las características del espacio doméstico, pueden ser influidos por este tipo de planteamientos.

En este ámbito se centran nuestros estudios primarios. Estos abordan una amplia variedad temática con ilazón argumental que comprende desde el análisis de la distribución diferencial de yacimientos arqueológicos a una escala de síntesis (realizado con el objetivo de componer mapas de comarcalización arqueológica de Galicia), hasta los análisis de emplazamiento de los yacimientos de diferentes momentos crono-culturales (realizados con el objetivo de reconstruir las estrategias de apropiación de la naturaleza y, a partir de ello, los patrones de racionalidad socio-cultural vigentes en cada uno de esos momentos). En esta misma línea abordamos el estudio de la cultura material arqueológica desde la perspectiva de la ArPa; aunque ello puede parecer contradictorio, en este caso lo que se pretende es examinar hasta qué punto los patrones de producción de la cultura material reproducen los mismos esquemas de racionalidad espacial presentes en otras escalas de las formaciones económico-sociales consideradas. 


\section{Evaluación}

La valoración patrimonial, la transformación del registro en Patrimonio Arqueológico, y los criterios de selección que esta operación implican, pueden ser orientados y fundados sobre la ArPa. La consideración de los elementos arqueológicos en el seno de un paisaje más amplio y en relación con el registro en él existente, permitirá establecer su valor relativo y definir la novedad y unicidad de un determinado elemento en comparación con los demás.

En nuestro caso concreto, la resolución de problemas prácticos como la evaluación y corrección de impacto arqueológico, se acomete desde esta perspectiva. Tiene la ventaja de que, dentro de una disciplina y problemática que son fundamentalmente subjetivas, la ArPa ofrece un marco que permite objetivar el proceso evaluador a partir de referentes externos y explícitos.

\section{Revalorización}

Los planes de rentabilización socio-cultural del Patrimonio Arqueológico también se pueden basar en la ArPa. Esta ofrece tanto un argumento o narrativa que hace accesible al público los elementos de ese Patrimonio, como recursos concretos (y económicos) para ponerlos en valor, además de una base práctica muy rentable constituida por el paisaje o paraje arqueológico-histórico-natural que los contiene.

Todo ello permite diseñar soluciones que, a través de la integración del Patrimonio Natural y el Histórico, facilitan los trabajos de ordenación del territorio de una determinada zona, plantean vías de solución de los problemas de protección de ese Patrimonio, atienden a su exhibición y puesta en valor, y definen los criterios de uso, rentabilización y, en su caso, consōlidación de los restos arqueológicos.

\section{Mercantilización}

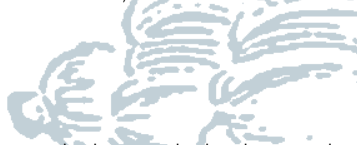

Teniendo en cuenta el crecimiento de la demanda de Turismo Rural y de calidad, así como el interés actual en su promoción y desarrollo, y la riqueza en bienes arqueológicos con la que cuenta nuestro país, es necesario y viable adoptar formas de rentabilización y puesta en valor del Patrimonio Arqueológico que convierta a éstos en recursos factibles de ser aprovechados como productos turísticos y de otro tipo.

\section{Sistematización}

Finalmente, las necesidades cada vez más acuciantes de disponer de inventarios ágiles, dinámicos e integrales de entidades arqueológicas, se pueden responder conjugando los planteamientos que emanan de la $\mathrm{ArPa}$, que permiten tratar la información arqueológica como datos geográficos, con el esquema valorativo y de toma de decisiones que se deriva de la cadena interpretativa. Sobre esta base se pueden diseñar y desarrollar sistemas de información que permitirían gestionar en todas sus dimensiones y con funcionalidad diferente re- cursos arqueológicos, y servirían como una herramienta básica de trabajo a cualquier grupo u organismo que necesite alimentar un Inventario complejo de Recursos Patrimoniales.

En nuestro caso, hemos diseñado y programado un Sistema de Información Arqueológica (SIA) para gestionar los datos generados por nuestros propios trabajos. Después de dos años de esfuerzo propio en este sentido, empezamos a utilizar la base disponible para desarrollar un Sistema integrado de gestión de Inventarios de Recursos Culturales que, de hecho, se encuentra ahora en fase de pre-comercialización. Este sistema no sólo permite gestionar información concerniente a yacimientos y entidades arqueológicas, sino también comprender los restantes ámbitos del Patrimonio Cultural (bienes etnográficos, históricos, arquitectónicos...)

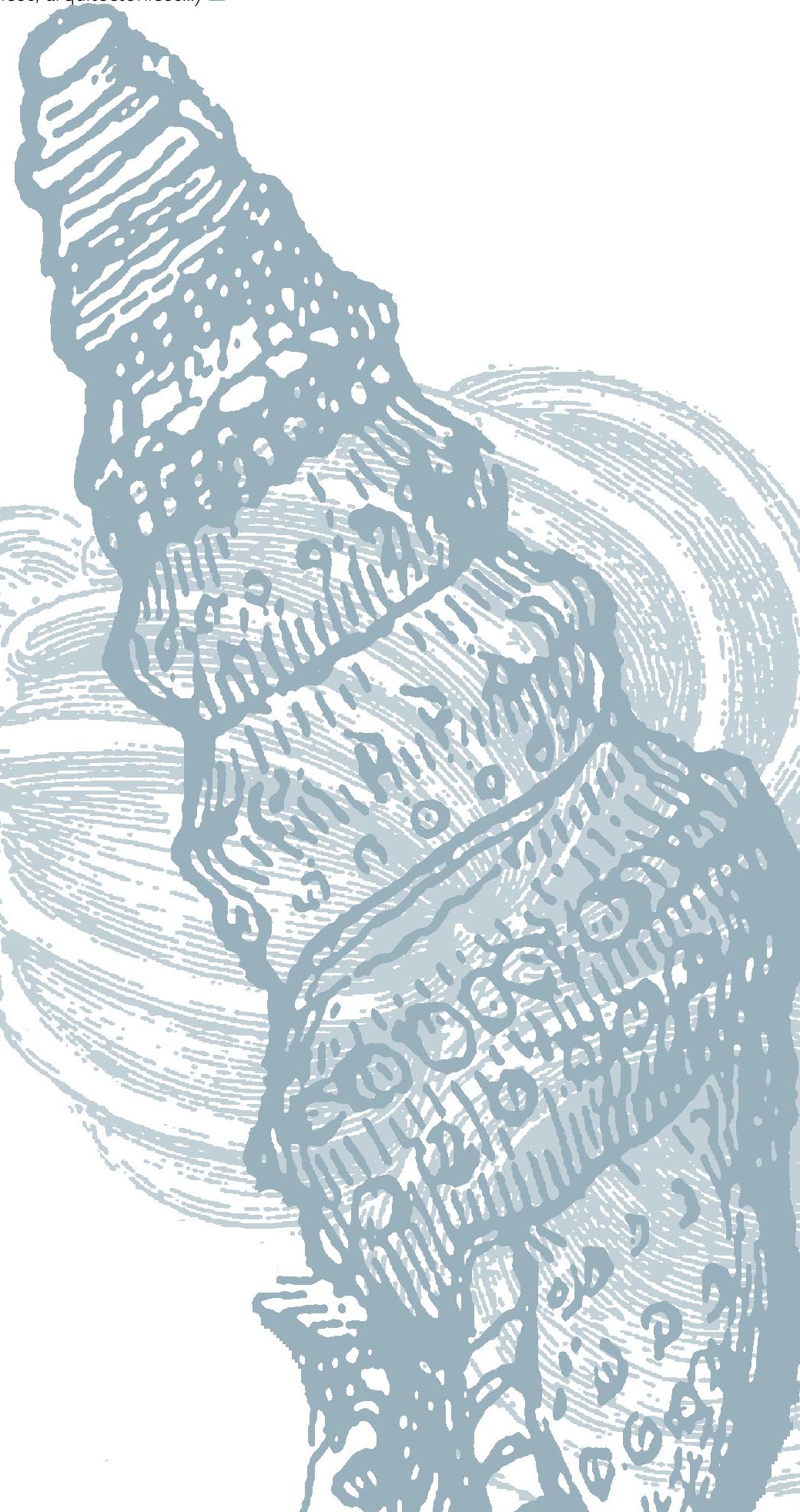

